\title{
Characterizing Ligand Interactions in Wild-type and Mutated HIV-1 Proteases
}

\author{
Leyte L. Winfield \\ Spelman College \\ Department of Chemistry \& \\ Biochemistry \\ 350 Spelman LN, SW, Box 231 \\ Atlanta, GA 30314 \\ Voice: (404) 270-5748 \\ Iwinfield@spelman.edu
}

\author{
Rosalind Gregory-Bass \\ Spelman College \\ Department of Biology \\ 350 Spelman LN, SW, Box 1183 \\ Atlanta, GA 30314 \\ RBass@spelman.edu
}

\author{
Jordan Campbell \\ Spelman College \\ Department of Chemistry \& \\ Biochemistry \\ 350 Spelman LN, SW, Box 231 \\ Atlanta, GA 30314 \\ j.campbell529@gmail.com
}

\author{
Andy Watkins \\ New York University \\ Department of Chemistry \\ 100 Washington Square East \\ New York, New York 10003 \\ andy.watkins2@gmail.com
}

\begin{abstract}
A computational module has been developed in which students examine the binding interactions between indinavir and HIV-1 protease. The project is a component of the Medicinal Chemistry course offered to upper level chemistry, biochemistry, and biology majors. Students work with modeling and informatics tools utilized in drug development research while evaluating wild-type and mutated forms of the HIV-1 protease in complex with the inhibitor indinavir. By quantifying the molecular interactions within protease-inhibitor complexes, students can characterize the structural basis for reduced efficacy of indinavir.
\end{abstract}

\section{Categories and Subject Descriptors}

K.3.1 [Computer Uses in Education -Computer-assisted instruction (CAI)]

\section{General Terms Measurement}

Human Factors, Measurement

\section{Keywords}

HIV, molecular modeling, indinavir, computational lab, drug resistance, mutations, protease, ligand interactions, cheminformatics

\section{INTRODUCTION}

Permission to make digital or hard copies of all or part of this work for personal or classroom use is granted without fee provided that copies are not made or distributed for profit or commercial advantage and that copies bear this notice and the full citation on the first page. To copy otherwise, or republish, to post on servers or to redistribute to lists, requires prior specific permission and/or a fee. Copyright CJOCSE, a supported publication of the Shodor Education Foundation Inc.

\subsection{Computer Aided Drug Design in the Un- dergraduate Curriculum}

Computers have been utilized throughout chemistry and biology curricula to visualize molecular information and simulate fundamental concepts. To this end, visualization and simulation exercises have been useful for introducing undergraduates to biomolecular interactions associated with drug activity. Early examples of such activities involved the use of Microsoft Excel to conduct quantitative structure activity relationship (QSAR) analysis [1]. Recent efforts have focused on the use of supercomputing, molecular modeling software, and informatics tools to allow students to see the benefit of such technologies in explaining biological processes such as protein function as well as the molecular rationale for drug action [2-6]. Two specific examples allow undergraduate students to visualize the conserved regions of selected kinases and observe the impact of site-directed mutagenesis $[7,8]$. In addition, computational modules have been developed for the graduate and K12 curriculums as well. In the graduate curriculum, computational tools have been used to assist pharmacy students understand drug-receptor interactions using Pymol and the Keele Active Virtual Environment (KAVE) [9, 10]. However, students primarily observed the three-dimensional molecules assigned by the instructor and were provided with little opportunity to explore the function of the software. In the K-12 classroom, learning strategies involving molecular visualizations have been used to address the relevance of biomolecules to everyday life [11].

The preceding examples focus primarily on visualizing biological target. An activity more directly related to what is considered rational drug design and development involves assessing the efficacy of inhibitors for various diseases using Autodock and Pymol [12]. The pharmacological aspects of drug action (i.e. drug absorption, metabolism, and excretion-ADME) where exploited by Kim et. al. who implemented a computational experiment in which the physiochemical parameters of known drugs were utilized to predict the ability of the drugs to cross the blood-brain 
barrier [13]. Similar to this, undergraduates completing a medicinal chemistry course in Australia created mathematical models that demonstrate the relationship of the known activities of adrenoceptor ligands to their calculated physicochemical properties [14].

The HIV-1 module presented here represents an option for introducing multiple aspects of computer-aided drug design and related software tools. The module goes beyond the visualization of biomolecular structures and simulation of their function, the primary focus of many of the published computational activities. The module allows students to calculate physiochemical parameters of indinavir, Figure 1, to gain insight into its binding interactions with the wild-type and mutated forms of HIV-1 protease. Basic drug design terminology was assessed via a quiz and rubric graded reports. A survey was administered to assess the success of the module.

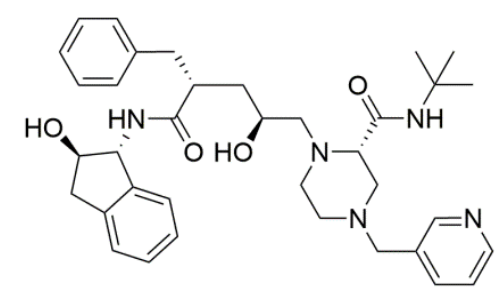

Figure 1: Indinavir

\subsection{HIV-1 Protease}

The HIV-1 protease is a key modulator of the HIV lifecycle [15, 16]. The proteolytic enzyme, comprised of 99 amino acid residues, functions to cleave the mature virus leading to its activation. Its activity is characterized by a base mediated amide hydrolysis that is catalyzed by two aspartate residues, ASP 25 and ASP 25'. The anionic form of the aspartate residue is required for the reaction to occur. Based on the mechanism shown in Figure 2, the active conformation of the enzyme is stabilized by a tetrahedrallike transition state [17]. Note that Brik and Wong provide a more acceptable mechanism that is concerted. However, the electron pushing scheme is not clearly delineated and doesn't coincide with what is understood at the undergraduate level. Protease inhibitors contain polar groups that facilitate van der Waals interactions within the active site of the enzyme, allowing the inhibitor to mimic the binding of the natural substrate and stabilize the transition state [18]. Such interactions prevent the enzyme from hydrolyzing the precursor polypeptides. Therefore, inhibiting the protease prevents the activation of the retrovirus and subsequent spread of HIV. Like other protease inhibitors, indinavir acts as a peptidomimetic. The molecule binds to the protease by forming a water mediated interaction to Ile50 and Ile50', inducing the active conformation of the enzyme and preventing the protease from interacting with its natural substrate.

\subsection{Indinavir}

Indinavir is one of nine drugs, not counting fosamprenavir which is hydrolyzed in the stomach to form amprenavir, licensed for the treatment of HIV-1 [19]. Despite the success of treatments with drugs like indinavir, HIV is a disease that quickly adapts to ensure its survival resulting in therapeutic resistance to many drugs. In the case of indinavir, its effectiveness against HIV-1 can be reduced due to mutations of only one amino acid residue. The mutation alters the conformation of the protease and its interac- tions with indinavir [20]. This module allows students to explore the three-dimensional structure of indinavir in complex with various mutated forms of the HIV-1 protease. Students utilize available X-ray data to observe the conformational differences between three indinavir-HIV-1 protease complexes (wild-type and two mutant complexes), gaining insight into the molecular characteristics of drug resistance. In addition, students are introduced to molecular modeling tools commonly used in the field of medicinal chemistry.

\section{SYSTEM REQUIRMENTS \\ 2.1 Hardware Requirements and Software Installation}

Tools found in the Molecular Operating Environment (MOE, Chemical Computing Group, http://www.chemcomp.com/) software, were utilized to accomplish modeling and computational tasks. The software is available free of charge for institutions that have an academic research license which costs approximately $\$ 2,500$ annually. However, the exercise can be adapted for use with other software including Sybyl (http://tripos.com/index.php) and Maestro/MacroModel (http://www.schrodinger.com/) which range in price. Some aspects can also be completed using Pymol (http://pymol.org/educational/) and MarvinSpace with appropriate calculator plugins (http://www.chemaxon.com/products/). The latter two are limited in their ability to manipulate the threedimensional structure of the protease and measure binding energies as describe herein, but are free for academic use. Instructions for completing the module using MOE, Maestro/MacroModel, and Pymol are included in the appendix. However, the results reported here were generated using MOE. The software, distributed to the students on USB flash drives along with license keys, runs on both Windows (XP, Vista, or Windows 7) and Mac OS X (10.5 or higher) operating systems. A three-button mouse is strongly suggested for manipulating the controls in the software; laptop users should use an external mouse as the mouse pad will not provide the needed functionality. MOE operates using a server-based license and is only functional on systems with access to the server. Despite the limit of mobile use, the automated functions (structure rendering, sequence alignments, energy minimization, protonation, etc.) make the software ideal for introducing informatics and computational methodologies to students.

\subsection{Molecular Structure Files}

The known three-dimensional structures of wild-type and mutant proteins and their ligands can be obtained from the Research Collaboratory for Structural Bioinformatics (RCSB) Protein Data Bank (PDB, http://www.rcsb.org/pdb/home/home.do). The X-ray data of the co-crystallized protease-ligand complexes used in this module were PDB codes 1SDT (wild-type), 1SDU (L90M mutant), and 1SDV (V82A mutant). The binding energies and inhibitory activity of indinavir in each complex were obtained from the RCSB Protein Data Bank as well. With the exception of adding protons, all computational analyses are based on the conformations of the complex imported from the RCSB Protein Data Bank.

\section{DESCRIPTION OF COMPUTATION- AL MODULE 3.1 Course Description}


Figure 2: Proposed mechanism and tetrahedral-like transition state for the proteolytic activity of the HIV-1 protease.

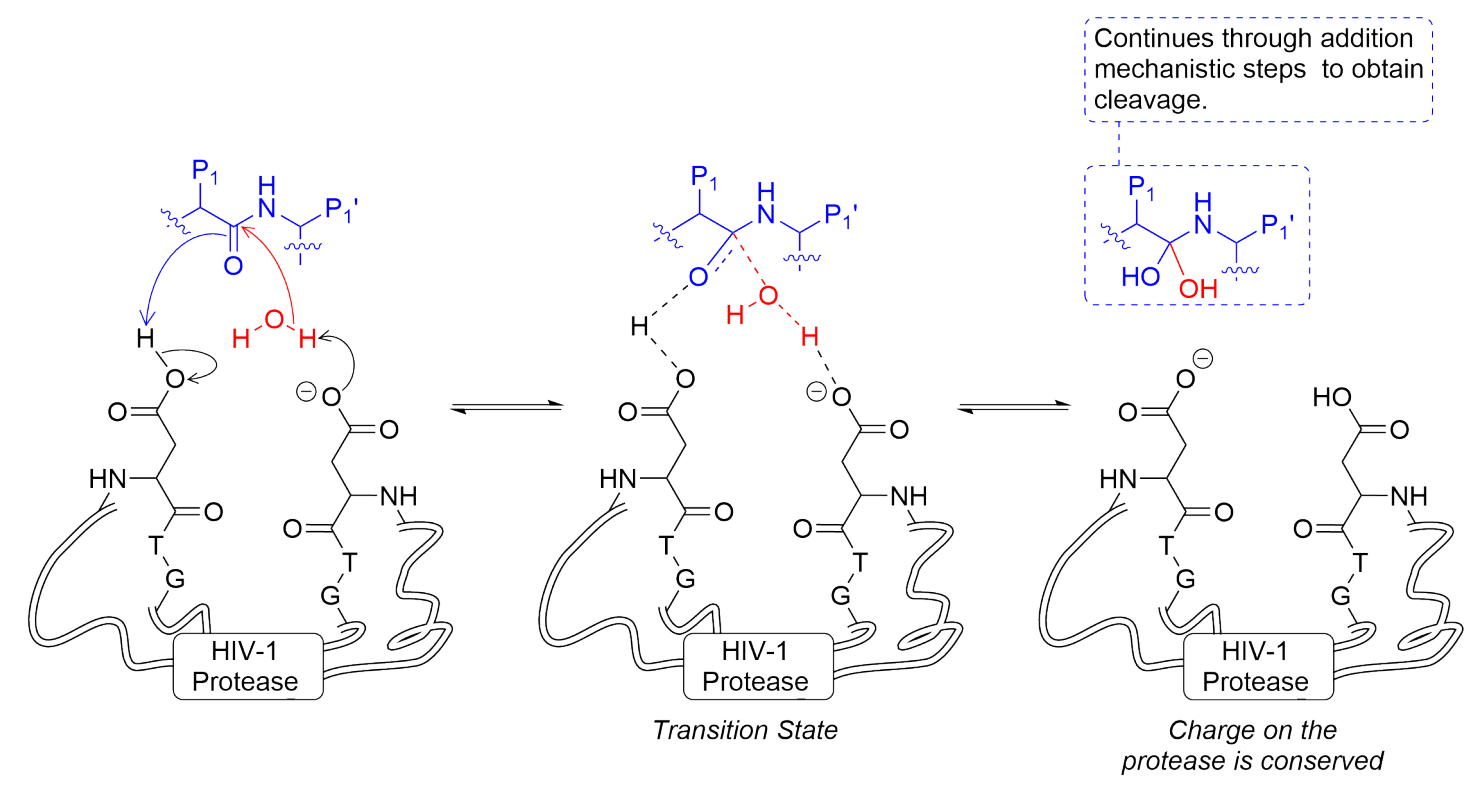

To date, the HIV module has been completed by three cohorts of students (average cohort size $=7$ ). The module is a component of the Medicinal Chemistry course, a one semester advanced elective offered to junior and senior chemistry, biochemistry, and biology majors. The course introduces students to computer-aided drug design (CADD) and cheminformatics tools utilized to characterize the molecular aspects of the disease. The course follows a blended format in which student led discussions and computational modules are the primary modes of instruction. The discussions are moderated by the instructor to ensure the correct information is being shared and to interject additional information as needed. The discussions are used to share information on the history of compounds with medicinal properties; the political, economic, environmental, and scientific frameworks for defining diseases; and the key concepts associated with drug design. Students are required to review information from the New York Times, scientific journals, and the Howard Hughes Medical Institute (HHMI) website in preparation for the discussions. For the computational components of the course, the instructor provides a brief overview on the purpose of the project and mini tutorials on the use of relevant software and databases.

\subsection{Module Structure}

The HIV module is completed over three weeks, each week containing two 2-hour class periods. The time was used for data collection and discussion of background information and results. Before beginning the computational component, students completed a literature search to identify background information and define key terms related to HIV/AIDS. Students were also required to watch the "HIV Lifecycle" and "Protease Inhibitors" found on the HHMI website $[15,16]$. In addition, students were provided with information on the mechanism which leads to the photolytic activity of the molecule, described in the introduction. Students were asked to analyze indinavir in complex with the wild-type HIV-1 protease and the two mutated proteases. The L90M (non-binding site mutation) and the V82A (binding site mutation) complexes were utilized. For the purpose of this assignment, it is sufficient for students to explore one mutated complex, but both should be examined if time permits. The mutated complexes are compared to the wild-type complex to understand how conformational changes reduce drug affinity and potentially reduce drug efficacy.

\subsubsection{Examining Binding Site Interactions}

Using the LigX function, protons were added to the threedimensional structure of each inhibitor-protease complex and the overall conformation was optimized (root mean square deviation, RMSD, of the final conformations were $<0.2 \AA$ from the original ligand in each case). The software assigns ionization states and adds hydrogens to structures based on the steric environment and protonation state of the chemical groups [21]. Standard force field parameters defined by Merck Molecular Force Field (MMFF94) were used to calculate the potential energy of the inhibitor and its affinity for the protease. The method is typically applied to calculations involving small molecules and provides suitable values for the type of comparative analysis conducted in this assignment. Key interactions between the ligand and the wildtype protease were identified and characterized as hydrogen bonding interactions (based on interaction distances and angles) or non-hydrogen bonding interactions. The cut-off for potential ligand-receptor interactions was set at $4.5 \AA$ for non-hydrogen bonding interactions with hydrogen bonding being defined at 1.4 $-2.2 \AA$. The in vitro binding energy $\left(\Delta G^{\circ}\right)$ for the indinavirprotease complex was provided to the students to define the relative ability of the ligand to bind to each complex in an energetically favorable conformation. Students also received data on the in vitro inhibitory activity of indinavir $\left(\mathrm{K}_{\mathrm{i}}\right)$.

\subsubsection{Comparing the Conformations of Indinavir}

The two protease files were opened in the MOE window. Each ligand structure was rendered in tube formula and given a distinct color. The conformations of the ligand-receptor complexes were then superposed. To accomplish this, the software treats the structures as rigid bodies and aligns the structures based on the threedimensional trace of the alpha helices [22]. The receptor was hidden to show the structural overlay of the two inhibitor conformations. Students measured the distance between the indane and benzene rings and the dihedral angle as defined by the pyridine 
and piperazine rings to quantify the differences between the two conformations. Students also examined the conformational differences between the wild-type and the mutated receptors by measuring intramolecular receptor interactions and the change in the water-mediated interaction between Ile50 and Ile50'. Finally, students identified the mutation present in their assigned complex and determined if the mutation occurred in the binding site of the protease.

\subsection{Evaluation}

Quiz questions, student reports, and survey responses were utilized as a preliminary gauge of the success of the module. All assessment materials were developed by the authors. The quiz contained a mixture of multiple choice and short answer questions created to determine students' basic concepts of drug design and HIV-1. The maximum score on each question was scaled to 1 point. Reports submitted by students were assessed using a rubric to determine their level of comprehension and ability to analyze the data collected in the completion of the module. The survey was administered through Survey Monkey at the completion of the course in years two and three of the module being offered. Part 1 of the survey contained fifteen questions that were posed to assess students' previous knowledge of drug design, attitudes towards course resources and technology, and future interest in research and drug design. Part 2 of the survey contained nine questions developed to measure students' confidence with the concepts and technology associated with the module. Anecdotal observations, gathered informally through class discussions and student reports, were documented to complement the survey results. The assessment of this module will be ongoing for future cohorts.

\section{RESULTS AND DISCUSSION 4.1 Computational Module}

Students submitted a report containing a summary of their results and selected images. The measurements obtained for the HIV-1 protease-indinavir complex along with the in vitro binding energies $\left(\Delta \mathrm{G}^{\circ}\right)$ of indinavir in complex with the protease and the concentration of indinavir needed to inhibit protease activity (inhibition constant, $\mathrm{K}_{\mathrm{i}}$ ) are given in Table $1[20,23]$. The energy of the interactions of indinavir with the protease, calculated binding energy (Table 1), was measured using standard force field parameters defined by Merck Molecular Force Field (MMFF94). The values were used by the students to characterize interactions that reduce the affinity of the drug for the protease. The calculated binding energies of indinavir in each complex correlate well with the experimental data $\left(\Delta \mathrm{G}^{\circ}\right.$ and $\left.\mathrm{K}_{\mathrm{i}}\right)$. There were cases in which students did not calculate the energies appropriately and did not see the expected trend. Students reporting this discrepancy were able to communicate it as an unexpected outcome. The number of students that did not correctly calculate the energies is accounted for in section 4.2.2.

Table 1. Sample of Student Data

\begin{tabular}{|c|c|c|c|}
\hline HIV-1 PR & $\begin{array}{c}\mathrm{K}_{\mathrm{i}} \\
(\mathrm{nM})\end{array}$ & $\begin{array}{c}\Delta \mathrm{G}^{\circ} \\
(\mathrm{kJ} / \mathrm{mole})\end{array}$ & $\begin{array}{c}\text { Calculated Binding } \\
\text { Energy } \\
(\mathrm{kcal} / \mathrm{mole}) \\
\end{array}$ \\
\hline Wild type & 0.60 & -54.75 & -12.14 \\
\hline mutant L90M & 0.80 & -54.01 & -11.78 \\
\hline mutant V82A & 1.34 & -52.68 & -11.55 \\
\hline
\end{tabular}

Students characterized the conformational changes of both the protease and the inhibitor that account for varied affinity towards binding to each protease. It should be noted for students that MOE classifies the $B$ chain of the protease different from the notation commonly used (i.e. Ile50' is annotated in the software as Ile150). The water mediated interaction between Ile50, Ile50', and indinavir (Figure 3) is believed to stabilize the active transition state of the protease. To calculate the angle of the interaction, hydrogen atoms were ignored. The angle, designated by the green dashed-lines in Figure 4, was defined using a nitrogen atom (of Ile50 or Ile50'), the oxygen of the water molecule, and a carbonyl oxygen of indinavir. This produced two water mediated interaction between indinavir and the protease. In the transition state described in Figure 2, the protease engages in a water catalyzed reaction with its natural substrate resulting in the cleavage of acyl bond. Inhibitors have been designed to bind to the protease in a similar fashion without a subsequent cleavage of the acyl bond. The interactions hold the protease in its active conformation and prevents the binding of the natural substrate. Therefore, the water-mediated interactions define an ideal orientation of Ile50 and Ile50' in the conformation of the protease when an inhibitor is present. The angles formed with Ile50' in the mutated proteases are wider than those formed in the wild-type protease, indicating the mutation causes a conformational change that prevents the inhibitor for occupying an optimal binding orientation when interacting with the protease. The change in the degree of the angle correlates well with the calculated affinities and the in vitro binding energies. Students identified the location of the mutation and noted that when the mutation occurred in the binding site (mutant V82A), the loss of affinity was more significant than when the mutation was at another point on the protease (mutant L90M). Based on the additional data collected (see supplemental documentation), students were able to elaborate on these observations in the context of drug resistance; in particular, how variations in the angles of these interactions could explain the differing activities of indinavir in the wild-type and mutated forms of the HIV-1 protease.

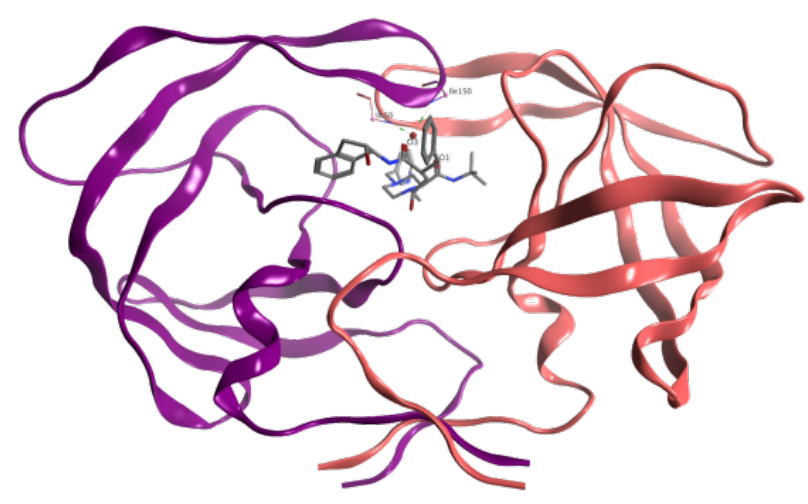

Figure 3: Relative Position of the Indinavir Binding Site in the HIV-1 Protease. Chain A in pink ribbon, Chain B in purple ribbon, indinavir grey tube formula, and protease residues in stick formula. 


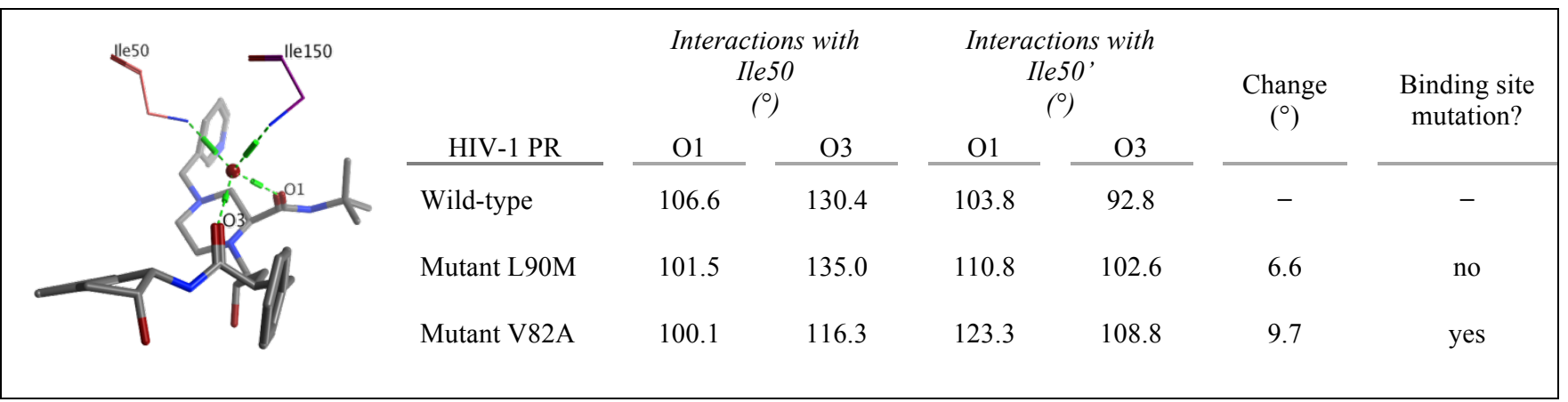

Figure 4. Ile50' (purple stick formula) and Ile50 (pink stick formula) interact with indinavir via a single water molecule, green dashed lines. The interaction is defined by an atom on the protease residues (the nitrogen of Ile50 or Ile50'), an atom from indinavir (one of the carbonyl oxygen atoms), and the oxygen of the water molecule (represented as a red ball). 01 and $\mathrm{O2}$ represent oxygen atoms 1 and 2, respectively, of indinavir in each complex. The average change is calculated as the difference is the angle between the atom of the wild-type protease for a given residue and that of the mutated proteases.

\subsection{Assessment and Evaluation}

\subsubsection{Evaluation of Student Learning}

To formally assess students' knowledge and comprehension of basic terminology, a quiz was administered. The assessment contained a mix of multiple choice and short answer questions (Table 1). In general, students' performed well on the quiz. The lowest performance was on the question of hydrogen bonding in drug action. Nevertheless, most students were able to elaborate on the need for electrostatic interactions to facilitate binding to the biological target. Assessment of student lab reports provided additional insight into students' conceptual understanding of the terminology. The reports were evaluated using a rubric which addressed the four questions given in Table 2. Students utilized information from assignments completed outside of class (literature search, assigned readings, and videos) and the group discussions to develop the background section of the reports. Most students were able to identify the appropriate literature and write an introduction that was relevant to the purpose of the assignment (Question 1). Although the transition state formed by the protease and its natural ligand was discussed and was the focus of the assignment, some students were unable to accurately describe its formation in their report (Question 2). Nevertheless, most students were able to collect accurate data and describe the correla- tion between the calculated binding energies and the actual binding energies or inhibitory activity (Question 3 ). In addition, students were able to correctly identify at least $80 \%$ of the ligand atoms and their corresponding binding interactions with the protease residues. Further, they were able to classify the interactions as water mediated or non-water mediated and identify the ligand atom as an H-bond donor or acceptor. This is especially important as hydrogen bonding is key to indinavir's ability to bind to the protease, as is the case for many protein-drug interactions.

\subsubsection{Evaluation of Course Structure}

A survey was used as a preliminary gauge of the benefits and success of implementing the module. Seven of the seventeen students from cohorts two and three responded to the survey. Part 1 of the survey (Table 3) addressed students' prior knowledge of course content, attitude towards the course, and future career goals and professional development activities. Most students indicated having little knowledge of drug design concepts or experience with computational modeling prior to the course. The module was designed to be a self-guided assignment, but the students received a tutorial from the instructor on how to work within the software. In addition, students could receive assistance from the instructor as needed, and were encouraged to work

Table 1. Post-Assessment of Student's Knowledge of Basic Terminology. Score (mean) represents the average score earned by students on a given question where $1=\mathbf{1 0 0} \%$.

\begin{tabular}{|c|c|c|}
\hline Question & $\begin{array}{c}\text { Score } \\
\text { (mean) }\end{array}$ & $\begin{array}{l}\text { Standard } \\
\text { Deviation }\end{array}$ \\
\hline 1. Excluding solubility as a reason, name two ways that H-bonding impacts drug activity & 0.50 & 0.47 \\
\hline 2. List the characteristics that describe the structure of a molecule. & 0.60 & 0.44 \\
\hline 3. Explain why we need new drugs & 0.80 & 0.42 \\
\hline $\begin{array}{l}\text { 4. Define syndrome and explain why HIV/AIDS was initially identified as a syndrome and not a } \\
\text { disease. }\end{array}$ & 1.00 & 0.00 \\
\hline 5. What type of intermolecular force is illustrated in the bonding interaction shown? & 1.00 & 0.00 \\
\hline 6. Which molecule has the best therapeutic index? & 1.00 & 0.00 \\
\hline 7. Define CADD. & 0.80 & 0.42 \\
\hline 8. Why is it important to evaluate mutation in drug targets? & 0.75 & 0.35 \\
\hline 9. Which molecule is most potent? & 0.90 & 0.32 \\
\hline 10. Which molecule is most efficacious? & 0.90 & 0.32 \\
\hline
\end{tabular}


Table 2. Assessment of Student Reports. Score (mean) represents the average score earned by students on a given question. Scores were assigned based on excellent $=4, \operatorname{good}=3$, fair $=2$, and poor $=1$.

\begin{tabular}{|c|c|c|c|}
\hline Question & $\begin{array}{c}\text { Score } \\
(\text { mean })\end{array}$ & $\begin{array}{c}\text { Score } \\
(\text { median })\end{array}$ & $\begin{array}{c}\text { Standard } \\
\text { Deviation }\end{array}$ \\
\hline $\begin{array}{l}\text { 1. Summary of background information related to the assignment including the } \\
\text { significance of the drug indinavir and the purpose/objective of the module. }\end{array}$ & 3.42 & 4.00 & 0.86 \\
\hline $\begin{array}{l}\text { 2. Summary of the mechanism of action for the HIV-1 protease and description } \\
\text { of related transition state. }\end{array}$ & 2.50 & 2.50 & 1.26 \\
\hline 3. Explanation of the correlation of the calculated energies to the known values. & 2.58 & 3.00 & 1.38 \\
\hline $\begin{array}{l}\text { 4. Identification of ligand- protease interactions (identify the interacting ligand } \\
\text { atoms as } \mathrm{H} \text {-bond donors or acceptors and protease residues) and the classifica- } \\
\text { tion of the interactions as water mediate or non-water mediate. }\end{array}$ & 2.67 & 3.00 & 1.31 \\
\hline
\end{tabular}

Table 3. Survey Part 1. Score (mean) represents students' self-reported level of agreement with each question, weighed on a scale of 0 to 4 (disagree $=0$, somewhat disagree $=1$, neither agree nor disagree $=2$, somewhat agree $=3$, and agree $=4$ ).

\begin{tabular}{|c|c|c|}
\hline Questions & $\begin{array}{c}\text { Score } \\
\text { (mean) }\end{array}$ & $\begin{array}{l}\text { Standard } \\
\text { Deviation }\end{array}$ \\
\hline 1. I knew about drug design before taking this course. & 2.14 & 1.77 \\
\hline $\begin{array}{l}\text { 2. I had taken a computational chemistry course or had conduct research in the area prior to taking } \\
\text { the course. }\end{array}$ & 1.86 & 2.04 \\
\hline 3. This course allowed me to analyze and interpret data; think critically. & 3.71 & 0.49 \\
\hline 4. This course challenged me to think and work "outside of the box." & 3.29 & 0.76 \\
\hline 5. It was useful to work with other students in a laboratory group. & 3.14 & 1.07 \\
\hline 6. The group discussions with the professor were useful. & 3.14 & 1.46 \\
\hline 7. The topics of this course were engaging. & 3.71 & 0.49 \\
\hline 8. I enjoyed the computational aspects of the course. & 2.57 & 1.81 \\
\hline 9. The course material provided sufficient detail to support self-guided and project-based learning. & 2.57 & 1.62 \\
\hline 10. The handouts were easy to follow. & 2.71 & 1.38 \\
\hline 11. The software was difficult to use. & 2.57 & 1.51 \\
\hline 12. I am now interested in research in the area of drug design and medicinal chemistry. & 3.14 & 1.46 \\
\hline 13. I may consider graduate school now. & 2.57 & 1.90 \\
\hline 14. This course is useful to students going to health professional or graduate school in STEM. & 3.86 & 0.38 \\
\hline 15. This course made me consider pursuing a summer research experience. & 3.43 & 1.51 \\
\hline
\end{tabular}

Table 4. Survey Part 2. Score (mean) represents students' self-reported level of confidence with concepts and technology related to the module, weighed on a scale of 4 to 1 (I am familiar with and understand the concept $=4$, I am familiar with and somewhat understand the concept $=3$, I am familiar with, but do not understand the concept $=2$, I am not familiar with the concept =1).

\begin{tabular}{|lcc|}
\hline \multicolumn{1}{|c}{ Question } & $\begin{array}{c}\text { Score } \\
\text { (mean) }\end{array}$ & $\begin{array}{c}\text { Standard } \\
\text { Deviation }\end{array}$ \\
\cline { 2 - 3 } 1. List the source of drugs & 3.00 \\
2. Explain the need for new drugs & 3.29 \\
\hline 3. Define CADD & 2.43 & 0.49 \\
\hline 4. Utilize the MOE software to draw 3D molecules & 2.86 \\
\hline 5. Utilize the MOE software to create a structural overlay & 2.43 \\
\hline 6. Utilize the MOE software to change the rendering of a protein and label residues & 1.21 \\
\hline 7. Utilize the MOE software to evaluate binding sites and ligand binding interactions & 2.57 \\
\hline 8. Describe the mechanism of action of protease inhibitors & 2.57 \\
9. Understand structurally what occurs when indinavir binds to the HIV-1 protease & 1.13 \\
\hline
\end{tabular}


collaboratively in completion of the work. Based on responses and informal conversations with students, key dynamics associated with access to and ease of use of the software must be considered. In informal conversations, students expressed resistance to only having access to the software while on campus. There were also difficulties with executing certain commands when students attempted to use the software on a Mac. There was also difficulty using the software without a three-button mouse. Therefore, convenience played a role in the students' level of intimidation and comfort with this project-based assignment. Nevertheless, most students found the module to be straight-forward and engaging once overcoming the initial difficulties of learning to use the software. One student stated, "This was an overall successful lab. The MOE software was difficult to understand at first. After the initial confusion, the data gathered from MOE was very interesting." Students had some difficulty with depth perception when looking at the three-dimensional structure, a difficulty that prevented them from being able to select the appropriate atoms when measuring distances and angles. Students also expressed difficulties manipulating the three-dimensional structure utilizing the various mouse functions to move and rotate the complex, which is not unusual for most individuals when first learning to work in a three-dimensional environment. Nevertheless, it initially caused students to have trepidation about completing the assignment.

In Part 2 of the survey, Table 4, students reported their level of understanding of the concepts. Across all questions, the majority of students reported a basic understanding of the concepts. Due to the small number of respondents, a formal statistical analysis was not performed. Since this is a preliminary assessment, interpretations of the outcomes should be cautious and cannot be generalized as a final measure of the success of this module. It is believed that exploring teaching strategies aimed at enhancing students' level of comfort with the software and decreasing intimidation with project-based learning will lead to the sustainability of this and other computational modules.

\section{Final Thoughts}

A major objective of the exercise was to introduce tools used in the design and virtual screening of medicinal molecules. The module illustrates for students how computational studies enhance the understanding of a drug's mechanism of biological systems. It is important to point out to students that computational models cannot stand alone in describing drug interactions, but should be used in context with experimental data. In a follow-up project, students might substitute the structural units of indinavir with an appropriate bioisostere/isostere and compare the interaction and energies of the modified structure to that of indinavir. Based on the theoretical results of such structural modifications, students can predict if the change would improve or reduces the affinity of the inhibitor for either of the HIV-1 proteases with respect to indinavir. Alternatively, students could mutate the protease further and determine the stability of the resulting indinavir-receptor complex in comparison to that of the wild-type protease. The procedure could be utilized to explore mutations and decreased drug effectiveness in Mycobacterium tuberculosis Protein Kinase B (MTB pknB), a Ser/Thr kinase which impacts cell growth and division in the disease. Doing so would expand the discussion of infectious diseases. Available PDB codes for this purpose are 1MRU, 3ORK, and 3ORK.

\section{ACKNOWLEDGEMENTS}

Contributions to and support for the development of this module was provided by the Faculty Resource Network and Dr. P. Arora, New York University (National Science Foundation Award No. CHE1151554 - Education Supplement). A.M.W. thanks New York University for the Kramer Fellowship. In addition, the HIV module is connected to a college-wide initiative at Spelman College to promote cross-divisional collaborations and interdisciplinarity. Drs. Marrionette C. Holmes and Shanie Harris are members of the team developing academic content related to HIV and AIDS.

This investigation has been funded in part by a grant from National Science Foundation Historically Black Colleges and Universities-Undergraduate Program (HBCU-UP) Grant Award No. 0714553, Advancing Spelman's Participation in Informatics Research and Education (ASPIRE), and National Science Foundation Award No. CHE1151554, Education Supplement. The content is solely the responsibility of the authors and does not necessarily represent the official views of the funding agencies listed.

There are no conflicts of interest to declare.

\section{REFERENCES}

[1] Muranaka, K. Anticancer activity of estradiol derivatives: A quantitative structure-activity relationship approach. $J$ Chem Educ, 78, 10 (Oct 2001), 1390-1393.

[2] Badotti, F., Barbosa, A. S., Reis, A. L. M., do Valle, I. F., Ambrosio, L. and Bitar, M. Comparative modeling of proteins: A method for engaging students' interest in bioinformatics tools. Biochem Mol Biol Edu, 42, 1 (Jan 2014), 68-78.

[3] Carvalho, I., Borges, A. D. L. and Bernardes, L. S. C. Medicinal chemistry and molecular modeling: An integration to teach drug structure-activity relationship and the molecular basis of drug action. J Chem Educ, 82, 4 (Apr 2005), 588-596.

[4] Hayes, J. M. An Integrated Visualization and Basic Molecular Modeling Laboratory for First-Year Undergraduate Medicinal Chemistry. J Chem Educ, 91, 6 (Apr 2014), 919-923.

[5] Roy, U. and Luck, L. A. Molecular modeling of estrogen receptor using molecular operating environment. Biochem Mol Biol Edu, 35, 4 (Jul-Aug 2007), 238-243.

[6] Rudnitskaya, A., Torok, B. and Torok, M. Molecular docking of enzyme inhibitors: A computational tool for structure-based drug design. Biochem Mol Biol Edu, 38, 4 (Jul-Aug 2010), 261-265.

[7] Chiang, H., Robinson, L. C., Brame, C. J. and Messina, T. C. Molecular mechanics and dynamics characterization of an in silico mutated protein: A stand-alone lab module or support activity for in vivo and in vitro analyses of targeted proteins. Biochem Mol Biol Edu, 41, 6 (Nov 2013), 402-408.

[8] Jones, M., Shoffner, R. and Friesen, J. Use of computer modeling of site-directed mutagenesis of a selected enzyme: A class activity for an introductory biochemistry course. $J$ Sci Edu Technol, 12, 4 (Dec 2003), 413-419.

[9] Richardson, A., Bracegirdle, L., McLachlan, S. I. H. and Chapman, S. R. Use of a three-dimensional virtual environment to teach drug-receptor interactions. Am J Pharm Educ, 77, 1 (Feb 2013).

[10] Satyanarayanajois, S. D. Active-Learning Exercises to Teach Drug-Receptor Interactions in a Medicinal Chemistry Course. Am J Pharm Educ, 74, 8 (Oct 2010). 
[11] Bethel, C. M. and Lieberman, R. L. Protein structure and function: An interdisciplinary multimedia-based guidedinquiry education module for the high school science classroom. J Chem Educ, 91, 1 (Jan. 2014), 52-55.

[12] Franco, D. T. a. J. Using Supercomputing to Conduct Virtual Screen as Part of the Drug Discovery Process in a Medicinal Chemistry Course. J Comp Sci Educ, 3, 2 (Feb 2012), 18-25.

[13] Kim, H., Sulaimon, S., Menezes, S., Son, A. and Menezes, W. J. C. A Comparative Study of Successful Central Nervous System Drugs Using Molecular Modeling. J Chem Educ, 88, 10 (Oct 2011), 1389-1393.

[14] Manallack, D. T., Chalmers, D. K. and Yuriev, E. Using the beta(2)-Adrenoceptor for Structure-Based Drug Design. $J$ Chem Educ, 87, 6 (Jun 2010), 625-627.

[15] HIV Lifecycle. HHMI BioInteractive Animation, City, 2007. http://www.hhmi.org/biointeractive/disease/hiv_life_cycle.ht $\mathrm{ml}$

[16] Protease Inhibitors. HHMI BioInteractive Animation, City, 2007.

p://www.hhmi.org/biointeractive/disease/protease_inhibitor.h tml

[17] Brik, A. and Wong, C. H. HIV-1 protease: mechanism and drug discovery. Organic \& Biomolecular Chemistry, 1, 1 (Jan 2003), 5-14.

[18] Drag, M. and Salvesen, G. S. Emerging principles in protease-based drug discovery. Nat Rev Drug Discov, 9, 9 (Sep 2010), 690-701.
[19] Ali, A., Bandaranayake, R. M., Cai, Y. F., King, N. M., Kolli, M., Mittal, S., Murzycki, J. F., Nalam, M. N. L., Nalivaika, E. A., Ozen, A., Prabu-Jeyabalan, M. M., Thayer, K. and Schiffer, C. A. Molecular basis for drug resistance in HIV-1 protease. Viruses-Basel, 2, 11 (Nov 2010), 25092535.

[20] Mahalingam, B., Wang, Y. F., Boross, P. I., Tozser, J., Louis, J. M., Harrison, R. W. and Weber, I. T. Crystal structures of HIV protease V82A and L90M mutants reveal changes in the indinavir-binding site. Eur J Biochem, 271, 8 (Apr 2004), 1516-1524.

[21] Labute, P. Protonate 3D: Assignment of Macromolecular Protonation State and Geometry. Chemical Computing Group Inc., City, 2007. http://www.chemcomp.com/journal/proton.htm

[22] Kelly, K. Protein Analysis in MOE: The Serine Proteases. Chemical Computing Group Inc, City, 2012. http://www.chemcomp.com/journal/triad.htm

[23] Vacca, J. P., Dorsey, B. D., Schleif, W. A., Levin, R. B., McDaniel, S. L., Darke, P. L., Zugay, J., Quintero, J. C., Blahy, O. M., Roth, E. and et al. L-735,524: an orally bioavailable human immunodeficiency virus type 1 protease inhibitor. Proc Natl Acad Sci U S A, 91, 9 (Apr 1994), 40964100 . 